\title{
A ENFERMAGEM E A EVOLUÇÃO DA IMPLANTAÇÃO DO PROGRAMA SAÚDE DA FAMÍLIA NO MUNICÍPIO DE MONTES CLAROS/MG: UMA ANÁLISE HISTÓRICO-DESCRITIVA (1994-2008)
}

\author{
Patrick Leonardo Nogueira da SILVA ${ }^{1}$ \\ Mariana de OLIVEIRA ${ }^{2}$ \\ Ana Augusta Maciel de SOUZA ${ }^{3}$ \\ Rogério Gonçalves da ROCHA ${ }^{4}$ \\ Tadeu Nunes FERREIRA ${ }^{5}$
}

\begin{abstract}
${ }^{1}$ Enfermeiro pelas Faculdades Integradas Pitágoras de Montes Claros/FIP-MOC. Especialista em Saúde da Família e Didática e Metodologia do Ensino Superior pela Universidade Estadual de Montes Claros/UNIMONTES. Pós-Graduando em Enfermagem do Trabalho pela Faculdade de Guanambi/FG. Guanambi (BA), Brasil. E-mail: patrick_mocesp70@hotmail.com

${ }^{2}$ Enfermeira pela FIP-MOC. Especialista em Saúde Pública pelas Faculdades Integradas de Jacarepaguá/FIJ e Didática e Metodologia do Ensino Superior pela UNIMONTES. Docente do Departamento de Enfermagem da Faculdade Santo Agostinho/FASA. Montes Claros (MG), Brasil. E-mail: marianamoc1 @ hotmail.com

${ }^{3}$ Enfermeira pela Universidade Federal de Minas Gerais/UFMG. Mestre em Ciências pela Universidade Federal de São Paulo/UNIFESP. Professora do Departamento de Enfermagem da UNIMONTES, FIP-MOC e FASA. Montes Claros (MG), Brasil. E-mail: anamaciel@ uai.com.br

${ }^{4}$ Enfermeiro pela UNIMONTES. Montes Claros (MG), Brasil. E-mail: rogeriorocha81@ @ahoo.com.br

${ }^{5}$ Enfermeiro pela UNIMONTES. Mestrando em Tecnologia da Informação pela Única Educacional/ÚNICA. Professor do Departamento de Enfermagem das Faculdades Unidas do Norte de Minas/FUNORTE. Montes Claros (MG), Brasil. E-mail: tadeu-nunes@ hotmail.com
\end{abstract}

Recebido em: 19/04/2015 - Aprovado em: 26/08/2015 - Disponibilizado em: 30/10/2015

\section{RESUMO}

Objetivo: analisar historicamente a evolução da implantação do Programa Saúde da Família como projeto estruturante e organizativo da atenção básica em Montes Claros/MG. Método: estudo histórico-descritivo, a partir de consulta nas bases da Biblioteca Virtual em Saúde (BVS) e Centro de Estudos e Pesquisa em Enfermagem da Secretaria Municipal de Saúde de Montes Claros (CEPEn-SMS-Moc) para a coleta dos títulos de periódicos de enfermagem e manuais da área de saúde pública do Ministério da Saúde, sendo as fontes provenientes de bibliotecas físicas e em bases digitais. Resultados: a busca resultou em 11 documentos publicados entre 1990 e 2009, sendo o primeiro trabalho de campo publicado em 2003. Considerações finais: no Brasil os sistemas de saúde eram orientados para a atenção especializada, reforçando-se o modelo hospitalocêntrico assistencialista. Este cenário vem sendo progressivamente modificado desde a introdução do Programa de Saúde da Família como estratégia de reorganização da atenção básica.

Palavras-chave: Artigo Histórico. Registros Eletrônicos de Saúde. Programa Saúde da Família.

\section{THE NURSING AND THE EVOLUTION OF THE DEPLOYMENT OF HEALTH PROGRAM FAMILY IN THE CITY OF MONTES CLAROS/MG: AN ANALYSIS OF HISTORICAL-DESCRIPTIVE (1994-2008)}

\begin{abstract}
Objective: to analyze historically the evolution of the implementation of the Family Health Program as a structural and organizational design of primary care in Montes Claros/MG. Method: study historical and descriptive, from consultation on the bases of the Virtual Health Library (VHL) and Centre for Studies and Research in Nursing Municipal Health Secretariat of Montes Claros (CEPEn-SMS-Moc) for collection of titles of periodicals nursing and manuals of public health from the
\end{abstract}


Ministry of Health, and the sources from physical to digital libraries and databases. Results: the search resulted in 11 documents published between 1990 and 2009, the first field work published in 2003. Final consideration: in Brazil health systems were targeted for specialized care, reinforcing the welfare hospital-centered model. This scenario has been progressively modified since the introduction of the Family Health Program as reorganization of primary care strategy.

Keywords: Historical Article. Electronic Records Health. Family Health Program.

\section{INTRODUÇÃO}

A implantação de programas de saúde que possuem uma hierarquização desde o nível federal ao municipal tem permitido tanto a elaboração de projetos que promovam o desenvolvimento local, quanto à utilização de conceitos e ferramentas inerentes à geografia no sentido de planejar a territorialidade de políticas públicas, de equipamentos e ações. O Programa Saúde da Família (PSF) foi proposto em 1994 como uma estratégia de reorientação do modelo assistencial, baseada no trabalho de equipes multiprofissionais em Unidades Básicas de Saúde (UBS). Estas equipes são responsáveis pelo acompanhamento de uma população adscrita, localizada em uma área delimitada, através de ações de promoção de saúde, prevenção, recuperação, reabilitação de doenças e agravos mais freqüentes. O PSF incorpora e reafirma os princípios básicos do Sistema Único de Saúde (SUS) e tem como desafio levar as práticas e ações de saúde de forma integral e contínua para mais perto da família e, com isso, melhorar a qualidade de vida dos brasileiros (BRASIL, 1997).

política plenamente sintonizada com os princípios da universalidade e da eqüidade da atenção e da integralidade das ações e, acima de tudo, voltado a permanente defesa da vida do cidadão (BRASIL, 2000, p. 9). Assim, desde 1999, passa a ser considerado pelo Ministério da Saúde (MS) como uma estratégia estruturante dos sistemas municipais de saúde, com vistas a reorientar o modelo assistencial e imprimir uma nova dinâmica na organização dos serviços e ações de saúde.

A mudança de denominação para Estratégia Saúde da Família (ESF) relaciona-se à necessidade de afirmação de sua proposta de gestão, organização e práticas de saúde como modos de operar os princípios e diretrizes do SUS, superando a idéia de programa, ou seja, de ações com tempo determinado para iniciar e finalizar (BRASIL, 2003). A ESF vem provocando um importante movimento na reordenação do modelo de atenção no SUS. 
Busca maior racionalidade na utilização dos demais níveis assistenciais e tem produzido resultados positivos nos principais indicadores de saúde das populações assistidas às equipes de Saúde da Família (SF) (BRASIL, 2008).

A ESF trabalha com a definição de território de abrangência, adscrição de clientela, cadastramento de famílias e acompanhamento da população da área. Prioriza as ações de prevenção, promoção e recuperação da saúde das pessoas, de forma integral e contínua, busca a complexa integração de ações individuais e coletivas, na manutenção da saúde desta comunidade, promoção da saúde, prevenção, recuperação, reabilitação de doenças e agravos mais freqüentes, com o firme propósito de propiciar o enfrentamento e a resolução dos problemas de saúde identificados na população adscrita (BRASIL, 2004; BRASIL, 2008).

$\mathrm{O}$ modelo preconiza uma equipe de SF de caráter multiprofissional (médico generalista, enfermeiro, auxiliar de enfermagem e agente comunitário de saúde [ACS]) trabalhando oito horas diárias e 40 horas semanais. A Unidade de Saúde da Família (USF) deve constituir a porta de entrada ao sistema local e o primeiro nível de atenção, o que supõe a integração à rede de serviços mais complexos. Recomenda-se que cada equipe fique responsável por entre 600-1000 famílias
(2400-4500 habitantes). A responsabilidade pelo acompanhamento das famílias coloca para as equipes de $\mathrm{SF}$ a necessidade de ultrapassar os limites classicamente definidos para a Atenção Básica $(\mathrm{AB})$ no Brasil, especialmente no contexto do SUS (BRASIL, 1999; BRASIL, 2008).

A ESF é um projeto dinamizador, condicionada pela evolução histórica e organização do sistema de saúde no Brasil. A velocidade de expansão da SF comprova a adesão de gestores estaduais e municipais aos seus princípios (BRASIL, 2008). O programa foi inicialmente divulgado pela representação da Fundação Nacional de Saúde (FUNASA) em Minas Gerais (MG) diretamente para os municípios, sem a participação da Secretaria de Estado de Saúde (SES). Em 17 de junho de 1994 a Comissão Intergestores Bipartite (CIB) Estadual aprova, através de deliberação, a inserção do PSF no estado, e partir de outubro o estado passa a conduzir o processo de implantação do PSF (VELOSO, 1995). Percebem-se na cultura da população brasileira, um excesso no consumo de serviços médicohospitalares, estes em geral de alta densidade tecnológica e elevado custo de produção. Muitos destes procedimentos são evitáveis, resolvíveis na Atenção Primária à Saúde (APS), na qual a população é atendida de forma 
coletiva, preventiva e promocional (BRASIL, 1997; BRASIL, 2000; BRASIL, 2003; BRASIL，2008; BRASIL，2004; BRASIL, 1999).

Desta forma, vê-se que os problemas que não são resolvidos na APS direcionam-se para determinar os custos sanitários e econômicos em outros pontos de maior densidade tecnológica da rede de atenção à saúde, assim, gerando ônus ao SUS. Uma das alternativas que as políticas públicas vêm procurando inserir nos municípios na tentativa de superar o paradigma dominante no campo da saúde é a ESF, que propõe a mudança na concepção do processo saúde-doença, saindo da oferta de serviços voltada para a doença para investir em ações de prevenção e promoção da saúde, reconhecendo a família como espaço para o desenvolvimento das ações (BRASIL, 1997; BRASIL， 2000; BRASIL， 2003; BRASIL, 2008; BRASIL, 2004; BRASIL, 1999; VELOSO, 1995).

Os serviços de saúde, quando bem orientados para a APS, são mais eficientes e possibilitam uma melhor utilização de outros níveis de atenção do sistema. Além disso, evitam internações desnecessárias; representam economia dos custos; melhoram a equidade; diminuem o uso de medicamentos; facilitam o controle público sobre o sistema; oferecem mais satisfação dos usuários e têm maiores impactos sobre os níveis de saúde (ARAÚJO, 1999).

Na APS o modelo de atenção à saúde, está centrado na família, entendida e percebida a partir do seu ambiente físico e social, o que possibilita, às equipes de SF uma compreensão ampliada do processo saúde/doença e da necessidade de intervenções que vão além de práticas curativas. O PSF é entendido como estratégia de reorganização do modelo assistencial dominante, e como especial alternativa para melhoria da APS (ARAÚJO, 2006).

O Governo de MG, no seu Plano de Governo e baseado na constatação de que o principal problema de Saúde Pública é a baixa qualidade da APS e dos serviços oferecidos, resolveu criar o Projeto Estruturador Saúde em Casa, como mais um incentivo financeiro para fortalecimento dos serviços na APS, e favorecimento da implantação e ampliação do PSF nos municípios. A evolução da ESF contextualiza-se diante do cenário nacional e do processo histórico-político de gestão pública, de forma a auxiliar na compreensão deste processo de organização da $\mathrm{AB}$ como redirecionador do modelo de assistência à saúde (ARAÚJO, 2006). 
Sendo assim, objetiva-se analisar historicamente a evolução da implantação do PSF como projeto estruturante e organizativo da $\mathrm{AB}$ em Montes Claros/MG.

\section{MATERIAIS E MÉTODOS}

Estudo bibliográfico, descritivo, na qual foi analisado e descrito a situação histórica de Montes Claros/MG desde o momento em que o PSF foi implantado, até os dias atuais, isto é, desde 1994, época de implantação do programa, até o momento atual nesta pesquisa, finalizando em 2008. Os dados foram obtidos junto aos arquivos da Secretaria de Municipal de Saúde (SMS) de Montes Claros, MG. Após a descrição da evolução histórica, foram pontuadas as possíveis questões no âmbito da gestão pública que possam estar envolvidas no processo de oscilação ou aumento do quantitativo de equipes contempladas pelo programa ao longo do tempo.

A revisão de artigos foi realizada através da consulta à Biblioteca Virtual em Saúde (BVS) e Centro de Estudos e Pesquisa em Enfermagem da Secretaria Municipal de Saúde de Montes Claros (CEPEn-SMS-Moc) considerado como critério inicial para seleção. Optou-se por estas bases de dados por serem as mesmas uma das principais fontes de publicações científicas na atualidade e, a partir de seu sistema de busca, utilizaram-se as seguintes palavras-chave: "Artigo Histórico"; "Registros Eletrônicos de Saúde"; e "Programa Saúde da Família”.

A coleta de dados pela base de dados física e eletrônica ocorreu no $1^{\mathrm{o}}$ semestre de 2010, entre os meses de março e abril do respectivo ano pelos próprios pesquisadores do trabalho. Para o mesmo, utilizou-se um formulário estruturado para a captação dos dados. A busca resultou em 11 documentos publicados entre 1990 e 2009, sendo o primeiro trabalho de campo publicado em 2003. Foi realizada uma leitura cuidadosa de todos os artigos selecionados, incluindo, neste estudo, aqueles que utilizaram métodos epidemiológicos na abordagem à implantação do PSF na cidade de Montes Claros, MG. Não houve a necessidade de submissão da presente pesquisa ao Comitê de Ética em Pesquisa (CEP), pois os dados estão publicados e de livre acesso. No entanto, o rigor metodológico durante toda a pesquisa foi contemplado.

\section{RESULTADOS E DISCUSSÃO}

De acordo com o MS, as mudanças no modelo assistencial, surgidas a partir de 1990, dentro do SUS, ocorreram principalmente por 
conta de dois programas: o Programa dos Agentes Comunitários de Saúde (PACS) e o PSF. Tais programas constituem importantes estratégias para a implantação das atividades de AB. Em 1988 foram introduzidas mudanças no funcionamento do Sistema de Saúde brasileiro com a criação do SUS. A implantação do SUS passa a ser efetivada a partir das Leis Orgânicas de Saúde (LOS), 8.080/90 e 8.142/90 e com as várias Normas e Portarias do MS, como instrumentos dinamizadores e de regulamentação do Sistema. Os princípios da universalidade para as ações de saúde, a descentralização para os municípios, a integralidade, a regionalização e a hierarquização, contidos na Constituição de 1988, definem a porta de entrada do serviço através do nível primário de atenção à saúde (BRASIL, 1990).

A ESF iniciou-se em 1994, ainda como programa. Foi condicionada pela evolução histórica e organização do sistema de saúde no Brasil, e vem apresentando crescimento expressivo nos últimos anos. Inicialmente como PSF, uma proposta de reformulação do PACS, trouxe a necessidade de incorporar novos profissionais ao processo de trabalho, para que os agentes não trabalhassem isoladamente. A concepção do PSF sempre foi a de fazer dele um instrumento de reorganização do SUS e da municipalização. Incorporou de áreas de risco para sua atuação a partir de estudos previamente elaborados, obedecendo também os princípios definidos pelo PACS quanto à seleção dos Agentes e a escolha a critérios como: interesse da comunidade, estudos de demanda, existência de serviços de apoio para referência dos usuários, entre outros. Estes programas provocaram alterações tanto nos padrões de financiamento das ações de saúde quanto na forma de organização dos serviços (DAL-POZ; VIANA, 1998).

Desde a implantação do programa em 1994, seu desenho já trazia em suas diretrizes a proposição de provocar mudanças no modelo assistencial para romper com a passividade dominante nas UBS, que até então só atendiam quem as procurava. As ações de saúde devem ser levadas para junto das comunidades e ser ao mesmo tempo coparticipantes do processo de cuidar e assistir (BRASIL, 1997). A SF é operacionalizada mediante a implantação de equipes multiprofissionais em UBS. Estas equipes são responsáveis pelo acompanhamento de um número definido de famílias, localizadas em uma área geográfica delimitada. As equipes atuam com ações de promoção da saúde, prevenção, recuperação, reabilitação de doenças e agravos mais freqüentes, e na manutenção da saúde desta comunidade (BRASIL, 2008). 
Considerando que tal reorientação é capaz de induzir a estruturação do SUS, a partir da organização da $A B$ nos sistemas municipais de saúde e do fortalecimento dos princípios de universalidade, acessibilidade, integralidade e eqüidade, o MS o vem denominando de ESF, considerando-o como o principal eixo de indução da identidade do modelo de atenção que se busca construir no SUS (BRASIL, 2003). A estratégia encerra, em sua concepção, mudanças na dimensão organizacional do modelo assistencial superando a antiga proposição de caráter exclusivamente centrado na doença, desenvolvendo-se por meio de práticas gerenciais e sanitárias, democráticas e participativas, sob a forma de trabalho em equipes, dirigidas às populações de territórios delimitados, pelos quais assumem responsabilidade (ESCOREL et al., 2007).

A estratégia do PSF está estruturada a partir da USF. Essa Unidade atua nos seguintes princípios (D’ALVA, 2004; BRASIL, 2008):

a) Caráter Substitutivo: não significa a criação de novas estruturas de serviços, exceto em áreas desprovidas, e sim a substituição das práticas convencionais de assistência por um novo processo de trabalho, cujo eixo está centrado na vigilância à saúde. Enfatiza o caráter substitutivo, enquanto opção de modelo de atenção, substituindo as unidades básicas tradicionais pelas USF;

b) Integralidade e hierarquização: a USF está inserida no primeiro nível de ações e serviços do sistema local de saúde, a AB. É a porta de entrada de um sistema hierarquizado e regionalizado de saúde, e deve estar vinculada a rede de serviços de forma a garantir atenção integral aos indivíduos e famílias, e a assegurar referência e contra-referência de forma articulada com todos os níveis do sistema, sempre que for requerida maior complexidade, a fim de garantir a integralidade e a resolutividade da atenção;

c) Territorialização e adscrição de clientela: trabalha com território de abrangência definido, e é responsável pelo cadastramento e acompanhamento da população adscrita a esta área. Cada equipe se responsabiliza pelo acompanhamento máximo de 4000 pessoas, sendo recomendada uma média de 3000 pessoas, que passam a ter co-responsabilidade no cuidado à saúde;

d) Equipe multiprofissional: a equipe de SF é responsável pela atenção à saúde de uma população adscrita, e institui novos profissionais à equipe, a saber, os agentes comunitários de saúde (ACS), voltados para a ação comunitária, ampliando a atuação sobre os determinantes mais gerais do processo saúde- 
enfermidade. A equipe de SF é composta minimamente por um médico generalista ou de família, um enfermeiro, um auxiliar de enfermagem e quatro a seis agentes comunitários de saúde. Quando ampliada, conta ainda com: um dentista, um auxiliar de consultório dentário e um técnico em higiene dental.

O trabalho de equipes da SF é o elemento-chave para a busca permanente de comunicação e troca de experiências e conhecimentos entre os integrantes da equipe e desses com o saber popular do ACS. O número de ACS na equipe varia de acordo com o tamanho do grupo sob a responsabilidade da mesma, que deve ser suficiente para cobrir $100 \%$ da população cadastrada, numa proporção de no máximo 750 pessoas por ACS, e até 12 ACS por equipe de SF. Os profissionais são vinculados a uma UBS e atuando numa área de abrangência definida, com clientela adstrita. A atuação das equipes ocorre principalmente nas UBS, no domicílio e na mobilização da comunidade pelos profissionais que compõem as equipes de SF, numa jornada de trabalho de 40 horas semanais (BRASIL, 1997).

A equipe de SF vem, portanto, com o desafio de pensar a família a partir do seu ambiente físico e social, com uma compreensão ampliada do processo de saúde/doença e de intervenções que vão além das práticas curativas, com perspectiva significativa na promoção da saúde de seus membros, trabalhando assim a unidade familiar e o meio onde ela vive. Nesse espaço da prática de $\mathrm{SF}$, o PSF assume também alguns compromissos importantes, tais como (BRASIL, 2000):

a) Entender a família e seu espaço social como núcleo básico da abordagem, não mais o indivíduo isoladamente;

b) Executar assistência integral, permanente, resolutiva, e de boa qualidade;

c) Intervir sobre os fatores de risco aos quais a comunidade está exposta e humanizar as práticas de saúde;

d) Incrementar conhecimentos interdisciplinares e realizar atividades de educação e promoção da saúde;

e) Criar vínculos de compromisso e de co-responsabilidade entre os profissionais de saúde e a comunidade;

f) Utilizar sistemas de informação para o monitoramento e a tomada de decisões;

g) Desenvolver ações intersetoriais, através de parcerias estabelecidas com diferentes segmentos sociais e institucionais, de forma a intervir em situações que transcendem a especificidade do setor saúde e que têm efeitos determinantes sobre as condições de 
vida e saúde dos indivíduos, famílias, comunidade;

h) Democratizar o conhecimento do processo saúde e doença, da organização do serviço e da produção social da saúde;

i) Reconhecer a saúde como um direito de cidadania e estimular a organização da comunidade para efetivo exercício do controle social das ações e serviços de saúde.

A partir de 1991, surge como tentativa de fortalecimento da $\mathrm{AB}$ o PACS, após resultados e experiências de diversas regiões do país com ACS e inspirado em experiências anteriores para prevenção de doenças através de informações e orientações sobre cuidados de saúde a grupos de riscos. O programa pode ser considerado o antecessor do PSF, pois enfoca a família como unidade de atenção de saúde, quebrando o paradigma de atenção programática para o indivíduo ao introduzi-lo na prática de prevenção e intervenção em saúde (BRASIL, 1994). O PACS, tendo como objetivo principal contribuir para a redução da mortalidade infantil e materna nas regiões Norte e Nordeste do país, através de extensão de cobertura dos serviços de saúde para áreas mais carentes e não assistidas, enfocou a família como uma unidade programática de saúde e não mais o indivíduo. O programa introduziu, também, a noção de área de cobertura por família (VELOSO, 1995).

As experiências acumuladas com os trabalhos realizados pelos ACS propiciaram ao próprio MS perceber que esses Agentes poderiam ser importantes na reorganização dos serviços básicos de saúde nos municípios. Em princípio, a escolha desses Agentes envolvendo desde o processo seletivo, a capacitação, o acompanhamento do seu trabalho, bem como as condições de gestão do município, a formação do Conselho Municipal de Saúde (CMS), os recursos humanos disponíveis, entre outros, foram questões relevantes para implantação do PACS. A partir do momento em que o município aderiu ao PACS como uma estratégia de ampliação de cobertura, com aprovação em suas instâncias colegiadas, este programa passou a ser mais um instrumento auxiliar na implantação do SUS e na reorganização da $\mathrm{AB}$ de saúde (DAL-POZ; VIANA, 1998). O PACS funciona como elo entre a população e os serviços de saúde através de atividades de promoção da saúde e de prevenção de doenças, identificando fatores determinantes do processo saúde-doença e contribuindo com a comunidade no cuidado com a saúde (BRASIL, 1994).

Atualmente, o PACS é considerado parte da SF. Assim como o PSF, tem como 
objetivo priorizar as ações de promoção, proteção e recuperação da saúde dos indivíduos e da família, independente da faixa etária, do estado de saúde, sempre de forma integral e contínua (CAZELLI, 2003). Os ACS podem ser encontrados em duas situações distintas em relação à rede do SUS: ligados a uma UBS ainda não organizada na lógica da SF; ou ligados a uma USF como membro da equipe multiprofissional (BRASIL, 2008).

O ACS é uma pessoa da própria comunidade, com vida igual à de seus vizinhos, mas que está preparado para orientar as famílias a cuidarem de sua própria saúde e também da saúde da comunidade. Ele age em sintonia com a unidade de saúde mais próxima. É um trabalhador que faz parte da equipe de saúde local. O ACS atende os moradores de cada casa em todas as questões relacionadas com a saúde: identifica problemas, orienta, encaminha e acompanha a realização dos procedimentos necessários à proteção, a promoção, a recuperação/reabilitação da Saúde das pessoas daquela comunidade. No PACS, as ações dos ACS são acompanhadas e orientadas por um enfermeiro/supervisor lotado em uma UBS. Os agentes do PACS estão vinculados na lógica ainda não organizada em SF, mas muitas vezes, em municípios onde há somente o PACS, este pode ser considerado um programa de transição para a SF (BRASIL, 2008; BRASIL, 1994; CAZELLI, 2003).

Segundo dados do MS, atualmente, encontram-se em atividade no país 204 mil ACS, estando presentes tanto em comunidades rurais e periferias urbanas quanto em municípios altamente urbanizados e industrializados (BRASIL, 2008). Os aspectos técnico-operacionais relativos aos princípios estabelecidos por essa Lei, a implantação das Normas Operacionais Básicas (NOB) do SUS desencadeou um processo de descentralização intenso, transferindo para os Estados e, principalmente, para os municípios, um conjunto de recursos e responsabilidades para a operacionalização do SUS (BRASIL, 2003). Os recursos de custeio da esfera federal destinados à assistência à saúde podem ser executados segundo duas modalidades: Transferência Regular e Automática (fundo a fundo) e Remuneração por Serviços Produzidos. As Transferências de Recursos Fundo a Fundo (Estaduais e Municipais) têm por base a habilitação dos municípios e Estados segundo as condições de gestão estabelecidas pela NOAS/SUS 01/02 (BRASIL, 2003).

Para monitorar as transferências de recursos para Estados e municípios, o Fundo Nacional de Saúde criou um Sistema de Transferências de Recursos Fundo a Fundo, 
cobrindo as transferências do Piso de Atenção Básica (PAB); Ações de Epidemiologia e Combate às Doenças e do Fundo de Ações Estratégicas e Compensação (FAEC). É importante lembrar que até 1997 não havia a subdivisão dos recursos transferidos para Estados e municípios, o que passou a ocorrer a partir de março de 1998 com a edição da Portaria 2.121/GM que implantou o PAB e separou o teto financeiro entre recursos para o financiamento da $\mathrm{AB}$ e para o financiamento da Assistência de Média e Alta Complexidade (BRASIL, 2003).

O PAB consiste em um montante de recursos financeiros federais destinados à viabilização de ações de $\mathrm{AB}$ à saúde e compõe o Teto Financeiro do Bloco da AB. Constitui-se no componente federal para o financiamento da $\mathrm{AB}$, sendo composto de uma fração fixa e outra variável. O PAB é composto de uma parte fixa (PAB fixo), destinada a todos os municípios, e de uma parte variável (PAB variável), que consiste em montante de recursos financeiros destinados a estimular a implantação das estratégias nacionais de reorganização do modelo de atenção à saúde: onde se inclui a SF, a Saúde Bucal (SB) e os ACS, dentre outros, como Compensação de Especificidades Regionais; Saúde Indígena; Saúde no Sistema Penitenciário (BRASIL, 2006).
Os repasses dos recursos dos $\mathrm{PAB}$ fixo e variável aos municípios são efetuados em conta aberta especificamente para essa finalidade, com o objetivo de facilitar o acompanhamento pelos Conselhos de Saúde no âmbito dos municípios, dos Estados e do Distrito Federal (DF) (BRASIL, 2006). Com o PAB, o SUS passou a dispor de uma forma de financiamento tendo por base transferências de recursos sob critério per capita e não mais sob o critério exclusivo de prestação de serviços. Essa nova forma de transferência de recursos constituiu um passo significativo para a construção de um sistema de saúde não restrito a um componente passivo de assistência médica, na medida em que o município passou a ter a responsabilidade de gestão de seus recursos, podendo alocá-los segundo a sua estratégia de integralidade das ações de saúde (BRASIL, 2003). Além disso, o critério per capita foi de fundamental importância para a redução das desigualdades regionais na distribuição dos recursos. $\mathrm{O}$ financiamento da $\mathrm{AB}$ se dará em composição tripartite.

a) PAB fixo: os recursos do $\mathrm{PAB}$ serão transferidos mensalmente, de forma regular e automática, do Fundo Nacional de Saúde aos Fundos MS e do DF calculado pela multiplicação de um valor per capita, fixado 
pelo MS, pela população de cada município e do DF definida pelo IBGE.

b) PAB variável: os recursos do PAB variável são parte integrante do Bloco da $\mathrm{AB}$ e terão sua utilização definida nos planos municipais de saúde, dentro do escopo das ações previstas nesta Política. O PAB variável representa a fração de recursos federais para o financiamento de estratégias nacionais de organização da $\mathrm{AB}$, cujo financiamento global se dá em composição tripartite. Para fazer jus ao financiamento específico do PAB variável da SF, dos ACS e da SB faz-se necessária alimentação obrigatória do Sistema de Informação da Atenção Básica (SIAB), cuja responsabilidade de manutenção e atualização é da gestão municipal.
Os valores dos componentes do $\mathrm{PAB}$ variável são definidos em Portaria específica pelo MS, são transferidos a cada mês, tendo como base o número de Equipe de Saúde da Família (ESF), Equipes de Saúde Bucal (ESB) e número de ACS, registrados no cadastro de equipes e profissionais do SIAB, e Cadastro Nacional de Estabelecimentos de Saúde (CNES) no mês anterior ao da respectiva competência financeira.

O município de Montes Claros/MG dispõe de uma ampla rede de saúde, dotada de profissionais altamente capacitados e equipamentos de primeira geração nos setores públicos e privados. A rede física municipal é dotada de 15 Centros de Saúde (CS) localizados na zona urbana (Quadro 1).

Quadro 1 - Distribuição da rede física municipal conforme os CS localizados na zona urbana. Montes Claros (MG), 2014.

\begin{tabular}{|l|l|}
\hline \multicolumn{2}{|c|}{ CENTROS DE SAÚDE (CS) } \\
\hline Bairro Antônio Pimenta & Bairro Planalto \\
\hline Bairro Cintra & Bairro Renascença \\
\hline Bairro Delfino Magalhães & Bairro São Judas \\
\hline Bairro Eldorado & Bairro Santos Reis \\
\hline Bairro Esplanada & Bairro Vera Cruz \\
\hline Bairro de Lourdes & Bairro Vila Oliveira \\
\hline Bairro Major Prates & Bairro Vila Sion \\
\hline Bairro Maracanã & \\
\hline
\end{tabular}

Fonte: Secretaria Municipal de Saúde/SMS. Montes Claros (MG), 2014.

Centros de Saúde da Família (CSF) instalados nas diversas regiões a seguir (Quadro 2). Os mesmos possuem em sua estrutura consultórios médicos, de enfermagem e odontológicos. 
Quadro 2 - Distribuição dos CS conforme região. Montes Claros (MG), 2014.

\begin{tabular}{|l|l|}
\hline \multicolumn{2}{|c|}{ CENTROS DE SAÜDE DA FAMÍLIA (CSF) } \\
\hline \multicolumn{1}{|c|}{ REGIÃO NORTE } \\
\hline CSF Cidade Industrial & CSF Vila Atlântida \\
\hline CSF Panorâmica (Zona Rural) & \multicolumn{1}{|c|}{ REGI Õ̃ SUL } \\
\hline \multicolumn{1}{|c|}{ REGIÃO SUDESTE } \\
\hline CSF Conjunto Habitacional José Carlos de Lima \\
\hline \multicolumn{1}{|c|}{ REGIÃO LESTE } \\
\hline CSF Santa Cecília & CSF Santa Rafaela \\
\hline CSF Independência II & $\begin{array}{l}\text { CSF Conjunto Habitacional Clarice Athayde } \\
\text { Vieira }\end{array}$ \\
\hline CSF Vila Anália & \\
\hline CSF Independência I &
\end{tabular}

Fonte: Secretaria Municipal de Saúde/SMS. Montes Claros (MG), 2014.

De acordo o Quadro 3, a rede física municipal localizada na zona rural é dotada de oito Postos de Saúde (PS) distribuídos em distritos:

Quadro 3 - Distribuição dos PS localizados na zona rural. Montes Claros (MG), 2014.

\begin{tabular}{|l|l|}
\hline \multicolumn{2}{|c|}{ POSTOS DE SAÚDE (PS) } \\
\hline Nova Esperança & Aparecida do Mundo novo \\
\hline Miralta & São João da Vereda \\
\hline Santa Rosa de Lima & Ermidinha \\
\hline São Pedro das Garças & Vila Nova de Minas \\
\hline
\end{tabular}

Fonte: Secretaria Municipal de Saúde/SMS. Montes Claros (MG), 2014.

Os CS foram capacitados para atender cerca de $50 \mathrm{mil}$ pessoas/mês, sendo 15 PS e três Policlínicas. Quanto ao atendimento odontológico, são realizados 40 mil procedimentos mensais, dos quais 2.420 são casos de urgência/emergência. A Prefeitura de Montes Claros contribui com cerca de $\mathrm{R} \$ 60.000$ reais para os Hospitais Santa Casa e Haroldo Tourinho, atenderem a comunidade nos casos de urgência e emergência. Foi implantado pela Secretaria Municipal de Saúde o atendimento noturno no PS do Bairro Santos Reis e nas Policlínicas Carlos José do Espírito Santo e Dr. Ariosto Machado. A Prefeitura adquiriu três ônibus médico-odontológico, totalmente equipados para atendimento na zona rural e bairros periféricos. O Programa "Acuidade Visual" leva médicos às escolas, 
onde os alunos com problemas de visão ganham óculos novos.

Quanto ao combate à desnutrição, 858 crianças com idade entre seis meses a um ano e 11 meses, ganharam leite gratuitamente. $\mathrm{O}$ objetivo é combater carências nutricionais. É distribuído leite integral, óleo de soja, sulfato ferroso e vitamina A e B. A Secretaria de Saúde, distribuiu 130 tipos de diferentes medicamentos da farmácia básica. Montes Claros possui hoje 108 dentistas nos CS, Policlínicas Municipais. Semanalmente 42 mil alunos fazem escovação acompanhada, e, com aplicação de flúor. A Prefeitura adquiriu um mamógrafo e um aparelho de ultra-sonografia para implantar um Programa de Prevenção de Câncer de Mama. O Programa de Acuidade Visual atendeu 34.454 crianças na faixa de cinco a 14 anos. Equipes médicas visitaram 8.422 pessoas nos bairros Vilage do Lago I e II, Vila Sion I e II, Alto da Boa Vista.

A seguir, são distribuídas por Policlínicas e Hospitais de Montes Claros (MG) conforme demonstrado no Quadro 4.

Quadro 4 - Distribuição da rede física municipal de atenção terciária conforme policlínicas e hospitais. Montes Claros (MG), 2014.

\begin{tabular}{|l|l|}
\hline \multicolumn{4}{|c|}{ POLICLÍNICAS } \\
\hline $\begin{array}{l}\text { Policlínica Carlos Espírito Santo - Bairro Alto } \\
\text { São João }\end{array}$ & $\begin{array}{l}\text { Policlínica Servidores Municipais - Ariosto } \\
\text { Correa Machado - Bairro Jardim Alvorada }\end{array}$ \\
\hline Policlínica Hélio Sales - Bairro Canelas & \multicolumn{3}{|c|}{ HOSPITAIS } \\
\hline $\begin{array}{l}\text { Fundação Hospitalar de Montes Claros / } \\
\text { Hospital Aroldo Tourinho }\end{array}$ & $\begin{array}{l}\text { Hospital Universitário Clemente de Faria / } \\
\text { HUCF-UNIMONTES }\end{array}$ \\
\hline $\begin{array}{l}\text { INCOR / Instituto de Cardiologia Aroldo } \\
\text { Tourinho }\end{array}$ & $\begin{array}{l}\text { Fundação Hospitalar Dilson de Quadros Godinho } \\
\text { / Hospital São Lucas }\end{array}$ \\
\hline $\begin{array}{l}\text { Irmandade Nossa Senhora das Mercês / Hospital } \\
\text { Santa Casa }\end{array}$ & Hospital UNIMED \\
\hline PRONTOCOR / Pronto Socorro do Coração & $\begin{array}{l}\text { PRONTOMENTE / Clínica Psiquiátrica de } \\
\text { Repouso }\end{array}$ \\
\hline
\end{tabular}

Fonte: Secretaria Municipal de Saúde/SMS. Montes Claros (MG), 2014.

O PSF foi implantado no ano de 1994, ano em que se iniciou em todo território nacional. As primeiras experiências de implantação do PSF ocorreram após a assinatura do Protocolo de Intenções entre o
MS e a SES em 24 de março de 1994, sendo o Dr. Henrique Santilho o então Ministro da Saúde, e o Dr. José Saraiva Felipe, o Secretário de Estado de Saúde de MG. O programa foi inicialmente divulgado pela Representação da 
FUNASA em MG diretamente para os municípios. Coube, nesta época, a esta Representação fazer as gestões administrativas junto aos municípios, sem a participação da SES, situação que causou alguns constrangimentos, quando então a Comissão Intergestores Tripartite (CIT) trouxe a si a questão para debate, passando à referida Secretaria a condução do processo de implantação do programa. Posteriormente, em 17 de junho de 1994, a CIB Estadual aprovou em deliberação a inserção do PSF no Estado, definindo fluxos e rotinas para a implantação do mesmo nos municípios. A partir de outubro, o Estado passa então a conduzir o processo de implantação do PSF (BRASIL, 2006).

Inicialmente, 12 municípios foram contemplados com recursos financeiros do MS, para a montagem de uma infra-estrutura mínima necessária à implantação do referido programa, completando um total de $40 \mathrm{ESF}$ e 288 ACS (BRASIL, 2006; MINAS GERAIS, 1994). Em janeiro de 1995, o PSF passa a fazer parte da Política de Saúde do Governo do Estado de MG como estratégia para reorganização da $\mathrm{AB}$. A partir dessa época, tem sido meta dos governos Federal e Estadual implantarem novas equipes do PSF e PACS com a finalidade de ampliar a cobertura populacional com assistência à saúde, priorizando as ações de promoção, prevenção de agravos e a oferta de ações curativas de menor complexidade (VELOSO, 1995). A Coordenação Estadual elaborou critérios técnicos e articulou-se com a Coordenação Nacional do MS para realizarem um trabalho que de fato propiciasse mudança na organização da $\mathrm{AB}$ de saúde nos municípios, e completou ao final de 1995, um total de 22 municípios e 42 equipes de SF (ARAÚJO, 1998).

A implantação no período de 1995 a início de 1996 ocorreu de forma lenta, dada as dificuldades identificadas na operacionalização do PSF nos municípios, tais como: desconhecimento dos gestores municipais dos objetivos finalísticos do PSF, que não entendiam o PSF como estratégia de organizar o modelo assistencial vigente, mas como uma nova alternativa de arrecadar recursos financeiros para o município; a inexistência de um sistema de referência e contra-referência para encaminhamento da clientela dentro do sistema proposto; presença de profissionais com formação voltada para a doença e especialidades, despreparadas para enfrentarem os problemas de saúde das comunidades; falta de treinamento introdutório para as equipes e gestores da área da saúde; grande rotatividade dos profissionais de saúde em busca de 
melhores condições de trabalho, em especial de salário; diferentes formas de contrato dos profissionais, gerando insegurança e, portanto, favorecendo a não fixação desses nos municípios (ARAÚJO, 1998).

Ainda no ano de 1996 houve um aumento substancial do número de municípios e ESF que aderiam ao PSF considerando a implantação do incentivo estadual como política pública. A partir de 1996 houve um aumento substancial do número de municípios e ESF que aderiam ao PSF considerando a implantação do incentivo estadual originado do Imposto sobre Circulação de Mercadorias e Prestação (ICMS). A partir de 1999, procedeuse a reorganização do PSF, bem como de sua ampliação em todo o Estado, corrigindo-se as distorções encontradas na constituição de muitas equipes, ou seja, das equipes que eram atestadas à SES como funcionantes.

A partir desse período, pela Portaria 1.329/99, estabeleceu-se a cobertura populacional das equipes de SF num determinado município como critério para definição do valor do incentivo financeiro, classificado em nove faixas distintas. Isso pode ter influenciado positivamente no incremento de equipes. Nesta época, após cinco anos de programa, estavam implantadas 868 equipes do PSF em 425 municípios mineiros, representando 49,82\% de cobertura dos municípios (ARAÚJO, 2001). No período de 2002 a 2008, a lei Hobin Hood foi um incentivo a mais para implantação de ESF nos municípios, uma vez que aqueles que adotassem o programa fariam jus receber o repasse do ICMS. Vale ressaltar que os valores de repasse são variáveis, já que dependem da arrecadação do Estado pelo ICMS e do número total de equipes implantadas. No período de 2002 a 2008, os recursos repassados pelo governo estadual pela Lei Hobin Hood representaram uma contribuição média mensal, por equipe, de $\mathrm{R} \$ 949,12$ aos municípios que atuassem com PSF (MINAS GERAIS, 2009).

Já se alcançaram valores mais expressivos, como em abril de 2008, tendo chegado a $\mathrm{R} \$ 1.326,82$ por ESF implantada. Mas atualmente, como o valor arrecadado pelo ICMS não tem aumentado na mesma proporcionalidade que o incremento de ESF, verifica-se que cada dia o quantitativo a ser repassado por equipe aos municípios tente a diminuir. De acordo com o valor de referência de janeiro de 2009, este repasse foi de apenas $\mathrm{R} \$ 767,73$ por equipe de SF (MINAS GERAIS, 2009). Em contrapartida ao baixo valor unitário de repasse, o valor total repassado aos municípios está aumentando, uma vez que estes vêm implantando maior número de ESF, e que 
esse montante varia de acordo com o número de equipes implantadas. Podemos perceber esta tendência ao compararmos os mapas de dois momentos pontuais do período - 2003 e 2007. Este caminhar do PSF trouxe avanços na oferta de serviços, porém foi pouco significativo na mudança de qualidade do atendimento.

Todas estas tentativas de incentivo do Estado de MG de elevar o quantitativo de equipes e fortalecer a qualidade da assistência na APS têm refletido positivamente na evolução do programa nos municípios mineiros. Nestes quinze anos da ESF, o Estado de MG ampliou muito o número de equipes $\mathrm{e}$ municípios cobertos pela estratégia. Atualmente, apenas 26 municípios mineiros ainda não possuem a $\mathrm{SF}$ como a principal estratégia de assistência à saúde (MINAS GERAIS, 2009; MINAS GERAIS, 2008; BOCK et al., 2010).

O Estado de MG é o Estado brasileiro com maior número de equipes. Os últimos dados do MS/Diretoria de Atenção Básica (DAB) se referem ao consolidado de 2007, e demonstram que MG contribuía com 12,94\% das equipes do país, destacando-se com 3.537 ESF no total de 27.324 ESF implantadas em território nacional (BRASIL, 2008). Já segundo dado mais recente, apurado pela SES em 2008, nota-se uma marca de 3.799 ESF. O quantitativo alcançado até dezembro de 2008 superou a meta de expansão esperada, que era de se alcançarem 3.700 equipes até o fim do ano. A meta almejada para o final de 2011 é de chegar a 4.000 equipes. Os dados considerados neste estudo para visualização do cenário estadual ao longo de quase quinze anos de PSF referem-se aos valores atestados mensalmente pelas Gerências Regionais de Saúde (GRS) à SES de MG (MINAS GERAIS, 2008; BOCK et al., 2010).

\section{CONSIDERAÇÕES FINAIS}

No Brasil, os sistemas de saúde eram orientados para a Atenção Especializada, reforçando-se o modelo hospitalocêntrico assistencialista. Este cenário vem sendo progressivamente modificado desde a introdução do PSF como estratégia de reorganização da $\mathrm{AB}$. O PSF, assim como o PACS, são estratégias e partes integrantes e fundamentais do SUS, que vêm avançando em todo o país, e de modo especial também nos municípios mineiros como em Montes Claros/MG. Este sucesso progressivo é verificado ao acompanharmos o quantitativo de equipes e número de municípios contemplados pelo programa ao longo dos anos. Alguns aspectos são fundamentais na evolução 
histórica do processo de implantação e de gestão em nível nacional e estadual que podem ter influenciado no caminhar do programa.

A implantação no período de 1994 a 1996 ocorreu de forma mais lenta, dada as dificuldades já identificadas anteriormente, que se configuram na operacionalização do PSF propriamente dita. Ou seja, era uma iniciativa ainda incipiente, desconhecida pelos gestores, sem uma rede que pudesse compor o referenciamento de casos, além do despreparo e rotatividade profissional, entre outras questões. A partir deste momento percebe-se um salto nos valores de equipes e municípios contemplados entre 1995 e 1996 e posteriormente um incremento progressivo. A partir de 1999 procedeu-se a reorganização do PSF, e sempre maior foi se mostrando o quantitativo de equipes nos municípios mineiros. Isso pode ser justificado, em parte, pelo aumento do incentivo federal ter passado a ser pago por cobertura populacional e em faixas distintas.

Desde o ano de 2005, tem se demonstrado um crescimento progressivo, porém mais linear. Isso pode ser visto como positivo se considerarmos estar ainda em ascensão, mas a linearidade também pode significar que estamos chegando a um ponto no qual não se obtêm maiores avanços no incremento de equipes, ou mesmo na cobertura de municípios com o programa, tendo ainda muito a melhorar. Pode significar talvez que a melhoria das condições do Programa deva ser então o principal foco, e que novas políticas de gestão pública precisam objetivar, mais veementemente, uma APS com SF de maior qualidade.

\section{REFERÊNCIAS}

ARAÚJO, M. R. N. SECRETARIA DE ESTADO DE SAÚDE DE MINAS GERAIS. GERÊNCIA DE ATENÇÃO PRIMÁRIA À SAÚDE. Projeto estruturador saúde em casa. Belo Horizonte: Ed. Mimeo; 2006.

ARAÚJO, M. R. N. SECRETARIA DE ESTADO DE SAÚDE DE MINAS GERAIS. GERÊNCIA DE ATENÇÃO PRIMÁRIA À SAÚDE. Programa saúde da família - PSF: princípios e diretrizes. Belo Horizonte (MG): Ed. Mimeo; 2001.

\section{ARAÚJO, M. R. N. A saúde da família: construindo um novo paradigma de intervenção no processo saúde-doença} [Tese]. São Paulo: Escola de Enfermagem da Universidade de São Paulo; 1999. 
ARAÚJO, M. R. N. O programa saúde da

família em Minas Gerais: saúde comunitária, atenção primária à saúde qualidade de vida. v. 2, n. 10, p. 10, 1998.

BOCK, L. F.; PADILHA, M. I.; VAGHETTI, H. H.; RAMOS, F. R. S. Produção de conhecimento na área de história da enfermagem no centro de estudos e pesquisa em enfermagem - ABEn (1972-2008). História da Enfermagem - Revista Eletrônica.

Brasília, v. 1, n. 2, p. 304-321, 2010.

BRASIL. MINISTÉRIO DA SAÚDE. DEPARTAMENTO DE ATENÇÃO BÁSICA. Programa saúde da família: atenção básica e a saúde da família. Brasília; 2008.

BRASIL. MINISTÉRIO DA SAÚDE. DEPARTAMENTO DE ATENÇÃO BÁSICA. Política Nacional de Atenção Básica - PNAB. Brasília; 2006.

BRASIL. MINISTÉRIO DA SAÚDE. DEPARTAMENTO DE ATENÇÃO BÁSICA. Monitoramento na atenção básica de saúde: roteiros para reflexão e ação. Brasília; 2004.

BRASIL. MINISTÉRIO DA SAÚDE. CONSELHO NACIONAL DE
SECRETÁRIOS DE SAÚDE. Para entender a gestão do SUS. Brasília: CONASS; 2003.

BRASIL. MINISTÉRIO DA SAÚDE.

Programa saúde da família: ampliando a cobertura para consolidar a mudança do modelo de atenção básica. Revista Brasileira de Saúde Materno Infantil. Recife (PE), v. 3, n. 1, p. 113-125, 2003.

BRASIL. MINISTÉRIO DA SAÚDE.

DEPARTAMENTO DE ATENÇÃO BÁSICA. Programa de saúde da família: a implantação da unidade de saúde da família. Cadernos de Atenção Básica. Brasília; 2000.

BRASIL. MINISTÉRIO DA SAÚDE. SECRETARIA DE ASSISTÊNCIA À SAÚDE. Manual para a organização da atenção básica. Brasília; 1999.

BRASIL. MINISTÉRIO DA SAÚDE. DEPARTAMENTO DE ATENÇÃO BÁSICA. Saúde da família: uma estratégia para a reorientação do modelo assistencial. Brasília; 1997.

BRASIL. MINISTÉRIO DA SAÚDE. FUNDAÇÃO NACIONAL DE SAÚDE. 
Programa de agentes comunitários de saúde:

normas e diretrizes. Brasília: FUNASA; 1994.

BRASIL. MINISTÉRIO DA SAÚDE.

Constituição da República Federativa do

Brasil 1988. Rio de Janeiro: Edições

Trabalhistas; 1990.

CAZELLI, C. M. Avaliação da implementação do Programa Saúde da Família no Município do Rio de Janeiro [Dissertação]. Rio de Janeiro: Escola Nacional de Saúde Pública Sérgio Arouca; 2003.

DAL-POZ, M. R.; VIANA, A. L. D. Reforma em saúde no Brasil: Programa de Saúde da Família. In: Estudos em Saúde Coletiva. Rio de Janeiro; 1998.

D’ALVA, M. G. M. Programa saúde da família na Guiné-Bissau: análise do processo de implementação na região sanitária de Bafatá [Dissertação]. Rio de Janeiro: Escola Nacional da Saúde Pública Sérgio Arouca; 2004.

MINAS GERAIS. FUNDAÇÃO JOÃO PINHEIRO. O histórico da lei Hobin Hood.

Belo Horizonte (MG): FJP; 2009.
MINAS GERAIS. SECRETARIA DE

ESTADO DE SAÚDE DE MINAS GERAIS. GERÊNCIA DE ATENÇÃO PRIMÁRIA À SAÚDE. Saúde em Casa. Belo Horizonte (MG); 2008.

MINAS GERAIS. SECRETARIA DE ESTADO DE SAÚDE DE MINAS GERAIS. GERÊNCIA DE ATENÇÃO PRIMÁRIA À SAÚDE. Relatório da Diretoria da Atenção Básica/PSF da Superintendência de Atenção à Saúde. Belo Horizonte (MG); 1994.

VELOSO, C. SECRETARIA DE ESTADO DA SAÚDE. GERÊNCIA DE ATENÇÃO PRIMÁRIA À SAÚDE. Relatório sobre o programa saúde da família em Minas Gerais. Brasília: Ed. Mimeo; 1995. 